\title{
Programmed death-ligand 1 positivity can predict improved survival and a lower risk of brain metastasis in patients with resectable small cell lung cancer
}

\author{
JIN LIU ${ }^{1}$, ZHONG LU ${ }^{2}$, WENHAO WANG ${ }^{1}$ and XIUMEI SUN ${ }^{2}$ \\ ${ }^{1}$ Department of Medical Oncology, Affiliated Hospital of Weifang Medical University; \\ ${ }^{2}$ Department of Oncology, Weifang Medical University, Weifang, Shandong 261000, P.R. China
}

Received December 12, 2017; Accepted April 16, 2018

DOI: $10.3892 / \mathrm{ol} .2018 .8895$

\begin{abstract}
The present study aimed to investigate the expression of programmed death-ligand 1 (PD-L1) in resectable small cell lung cancer (SCLC) and investigate its predictive value for survival and brain metastasis (BM). Postoperative SCLC specimens were immunostained with the SP142 antibody against PD-L1. Positive PD-L1 expression was defined as PD-L1 expression in $\geq 5 \%$ of tumor cells. A total of 80 patients were recruited between January 2010 and December 2012. PD-L1 was expressed in 65.0\% (52/80) of all patients and $59.3 \%(16 / 27)$ of patients with BM. The median survival time (MST) was longer in the PD-L1(+) group (46.4 vs. 28.5 months, $\mathrm{P}=0.002$ ). There was no significant difference in the MST between patients with PD-L1(+) or (-) $\mathrm{BM}(\mathrm{P}=0.55)$. The 3 -year risk of $\mathrm{BM}$ in the $\mathrm{PD}-\mathrm{L1}(+)$ group was lower than that in the PD-L1(-) group (24.1 vs. $48.4 \%$, $\mathrm{P}=0.046)$. PD-L1 was an independent factor for overall survival (OS) [hazard ratio $(\mathrm{HR})=0.485, \mathrm{P}=0.011$ ] and $\mathrm{BM}$ $(\mathrm{HR}=0.335, \mathrm{P}=0.024)$. The present study concludes that PD-L1 is commonly expressed in SCLC and is associated with OS and BM.
\end{abstract}

Correspondence to: Dr Xiumei Sun, Department of Oncology, Weifang Medical University, 7166 Baotong Western Road, Weifang, Shandong 261000, P.R. China

E-mail: 274881097@qq.com

Abbreviations: SCLC, small cell lung cancer; OS, overall survival; BM, brain metastasis; MST, median survival time; PCI, prophylactic cranial irradiation; PD-L1, programmed death ligand 1; HR, hazard ratio; MRI, magnetic resonance imaging; CT, computed tomography; PET, positron emission tomography; POCT, postoperative chemotherapy; PORT, postoperative irradiation; IHC, immunohistochemistry

Key words: programmed death-ligand 1, brain metastasis, small cell lung cancer, survival, prognostic factor

\section{Introduction}

Lung cancer has become the leading cause of cancer-associated mortality worldwide (1). Small cell lung cancer (SCLC) is the main subtype of lung cancer and accounts for $15-20 \%$ of all lung cancer cases (2). The incidence of cancer-associated mortality has increased from 28 to $50 \%$ among females with SCLC based on the surveillance, epidemiology and end results database (2). SCLC is characterized by a rapid growth rate and a positive response to treatment, but relapse often occurs (3). Consequently, the overall survival (OS) is poor, with a 5-year survival rate of $20 \%$ for limited-stage SCLC and $9.1 \%$ for extensive-stage SCLC, despite the sensitivity of SCLC to chemoradiotherapy $(4,5)$. The role of surgery in SCLC remains controversial, even though surgery could prolong survival in a subpopulation of individuals with SCLC (6). In addition, the risk of distant metastasis increases with prolonged survival. The brain is the most common organ of distant metastasis in patients with SCLC. The prevalence of brain metastasis (BM) ranges between 10 and $24 \%$ at the time of diagnosis and increases to $50 \%$ at 2 years post-diagnosis. Therefore, the prognosis of patients with BM is poor, with a median survival time (MST) of 4-6 months (7-9). Prophylactic cranial irradiation $(\mathrm{PCI})$ decreases the risk of $\mathrm{BM}$ but increases survival in patients with surgically resected SCLC, excluding pathological-stage I patients (10). PCI of unselected patients not only is a waste of medical resources but also negatively impacts patient quality of life with a low cost-efficacy. Therefore, an effective biomarker with high sensitivity or specificity that can predict BM in SCLC is urgently required.

Programmed death-ligand 1 (PD-L1, also known as B7-H1 or CD274) is present in a variety of tumor cells, including malignant melanoma, non-small cell lung cancer and SCLC (11-13). PD-L1 binds with PD-1 on tumor cells, competitively inhibiting the binding of B7 and CD28 and suppressing the ability of activated $\mathrm{T}$ cells to promote immune escape and tolerance $(14,15)$. A number of studies have investigated the distribution of PD-L1 in SCLC and investigated the correlation between PD-L1 expression and clinical outcomes, particularly in patients with BM (16-22). Increased expression of PD-L1 in a subset of SCLC tumors caused by focal amplification of CD274 suggested that the PD-L1 axis may be 
a novel therapeutic target for SCLC (23). A retrospective study revealed that PD-L1 was positively expressed in $51.8 \%$ of SCLC specimens $(n=83)$ and demonstrated that the MST was significantly longer in patients with PD-L1(+) tumors than in those with PD-L1(-) expression (17.0 vs. 9.0 months, $\mathrm{P}=0.018$ ) (20). Another retrospective study revealed that PD-L1 was highly expressed in SCLC (15-45\%) and that PD-L1(+) expression was correlated with improved disease-free survival, compared with PD-L1(-) expression [hazard ratio (HR) $=0.268, \mathrm{P}=0.003$ ] (16). Additionally, PD-L1 expression in tumor cells was observed in $75.0 \%(24 / 32)$ of BM specimens obtained from patients with SCLC (21). Furthermore, high levels of PD-L1 expression were negatively correlated with BM size, which indicated that anti-PD-L1 potentially reduced the incidence of BM (24). Based on the aforementioned evidence, PD-L1 expression may predict BM in patients with SCLC and targeting PD-L1 may prevent BM in patients with SCLC.

To date, the prevalence of PD-L1 expression in SCLC and the correlation between PD-L1 expression and BM in SCLC remains controversial. Therefore, a retrospective study was conducted to detect PD-L1 expression in postoperative SCLC specimens and to further investigate the role of PD-L1 expression in BM and OS. The results demonstrated that PD-L1 was highly expressed in SCLC $(65.0 \%, 52 / 80)$ and that PD-L1 expression was associated with improved OS and a lower risk of BM.

\section{Patients and methods}

Patients. Postoperative specimens were collected from 80 patients who had undergone complete resection between January 2010 and December 2012 at The Affiliated Hospital of Weifang Medical University (Shandong, China) and were stained with hematoxylin and eosin to identify SCLC cells. Prior to surgery, standardized evaluations were performed, including constant thoracic and abdominal computed tomography (CT), brain magnetic resonance imaging (MRI) and bone radionuclide imaging. Positron emission tomography (PET)-CT was performed on certain patients. All patients were staged according to the criteria of the American Joint Committee on Cancer (AJCC) 7th edition (25). Pathological node $0-2(\mathrm{pNO}-2)$ and p-stage I-III were used accordingly. Of the 80 patients, $45(56.3 \%)$ were male and $35(43.7 \%)$ were female. The median age for the whole cohort at diagnosis was 54 years, with a range of 34-72 years. Surgical procedures consisted of lobectomy or pneumonectomy with mediastinal nodal dissection. Postoperative chemotherapy (POCT, 4-6 cycles) with cisplatin/etoposide (EP) or carboplatin/etoposide (CE) was administrated. For postoperative thoracic irradiation (PORT), a total dose of 50-60 Gy was administered using a three-dimensional conformal radiotherapy or intensity-modulated radiotherapy technique for 5-6 weeks with 1.8-2.0 Gy per fraction for 5 days per week. For patients without BM identified by brain MRI prior to PCI, a total dose of 30 Gy with 3.0 Gy per fraction, or a total dose of $25 \mathrm{~Gy}$ with 2.5 Gy per fraction was administrated. PCI was delivered concurrently or sequentially with chemoradiotherapy.

The present study was approved by the institutional review board and ethics committee of the Affiliated Hospital of Weifang Medical University and Shanghai Changhai Hospital
(Shanghai, China). Written informed consent was obtained from every patient and/or their legal guardian prior to analysis.

Immunohistochemistry (IHC). Immunohistochemistry was performed to determine PD-L1 expression in resected specimens. Formalin-fixed (formalin concentration, 10\%; 12-24 h at room temperature), paraffin-embedded sections (4- $\mu \mathrm{m}$ thick) were air-dried at room temperature overnight. Sections were deparaffinized with $100 \%$ xylene $\left(25^{\circ} \mathrm{C}\right)$ and rehydrated through a grade ethanol series (100, 95 and $70 \%$ ethanol) at $100^{\circ} \mathrm{C}$ for $15 \mathrm{~min}$. Following this, antigen retrieval $\left(190^{\circ} \mathrm{C}\right.$ for $5 \mathrm{~min}$ ) was performed in a high-pressure cooker following washing of the slides 3 times with PBS. Peroxidase activity was quenched by $3 \%$ hydrogen peroxide at room temperature for $5 \mathrm{~min}$, then the tissues were incubated for $15 \mathrm{~min}$ in $5 \%$ fetal bovine serum at room temperature (Gibco; Thermo Fisher Scientific, Inc., Waltham, MA, USA) blocking buffer. As no antibodies were approved for NSCLC detection when the present study took place, the primary antibody SP142 (cat. no. 07309554001; 1:100; Spring Bioscience Corporation, Pleasanton, CA, USA) was utilized and incubated for $1 \mathrm{~h}$ at $25^{\circ} \mathrm{C}$, followed by incubation with the secondary HRP-labeled anti-rabbit antibody (cat. no. K4003; 1:100; Dako; Agilent Technologies, Inc., Santa Clara, CA, USA) for $30 \mathrm{~min}$ at room temperature. Following incubation in 3,3'-diaminobenzidine (cat. no. DAB-0031; Fuzhou Maixin Biotech Co., Ltd., Fuzhou, China), the slides were counterstained with hematoxylin $\left(15 \mathrm{sec}\right.$ at $\left.25^{\circ} \mathrm{C}\right)$ followed by dehydration and mounting with a coverslip. The Olympus BX51 light microscope was used to observe the staining status (magnification, $x 40$ ). In concordance with a previous study, human placenta (obtained from Changhai Hospital of Shanghai in July, 2014) was used as a positive control specimen for PD-L1 IHC (26). Written informed consent was obtained at the time the placenta samples were collected.

Evaluation of PD-L1 expression was performed by at least two pathologists who were blinded to the clinical data. PD-L1 expression at the cell membrane was evaluated by combining staining intensity and distribution scores. Staining intensity was scored as follows: 0 , no staining; $1+$, weak; $2+$, moderate and $3+$, strong. The final histoscore was formulated as follows: [weak (1+) x1] x percentage $+[$ moderate $(2+) \times 2] \times$ percentage $+[$ strong $(3+) \times 3]$ $x$ percentage. Weak $[1+] \times$ percentage refers to the percentage of tumor cells with weak staining. Moderate $[2+]$ /strong [3+] staining was also included into this formula. According to the references cited in this study, PD-L1 expression $\geq 1, \geq 5$ or $\geq 10 \%$ was mostly defined as PD-L1(+). However, no significant difference in survival was observed between the PD-L1(-) group and the PD-L1(+) group when PD-L1(+) was defined as PD-L1 expression $\geq 1$ or $\geq 10 \%$, respectively, therefore PD-L1(+) expression was defined as PD-L1 expression $\geq 5 \%$.

Follow-up. Patient follow-up occurred every 3 months for up to 2 years, every 6 months for the following 3 years and annually thereafter. The standard follow-up included a medical history and physical examination, chest CT, abdominal CT or ultrasound and bloodwork as clinically indicated. PET/CT and brain MRI were not routinely recommended, but for patients with suspected BM, an enhanced MRI or CT scan of the brain was performed. 
Table I. Clinical features of 80 patients with completely resected small cell lung cancer.

\begin{tabular}{lcc}
\hline Variable & $\mathrm{n}$ & $\%$ \\
\hline Sex & & \\
Male & 45 & 56.2 \\
Female & 35 & 43.8 \\
Age, years & & \\
$\geq 60$ & 26 & 32.5 \\
$<60$ & 54 & 67.5 \\
pN stage & & \\
N0-1 & 55 & 68.7 \\
N2 & 25 & 31.3 \\
p-stage & & \\
I & 25 & 31.3 \\
II & 23 & 28.7 \\
III & 32 & 40 \\
POCT & & \\
Yes & 62 & 77.5 \\
No & 18 & 22.5 \\
PORT & & 88.7 \\
Yes & 71 & 11.3 \\
No & 9 & 63.7 \\
PCI & & 36.3 \\
Yes & 51 & POCT \\
No & 29 & \\
\hline
\end{tabular}

$\mathrm{pN}$, pathological lymph node; p-stage, pathological stage; POCT, postoperative chemotherapy; PORT, postoperative radiotherapy; PCI, prophylactic cranial irradiation.

Statistical analysis.SPSS 22.0 statistical analysis software(IBM Corp., Armonk, NY, USA) was used to analyze all data in the present study. A $\chi^{2}$ test was performed to compare the patients' clinical characteristics. Measurement data and enumeration data are presented as median number and percentage (\%), respectively. All statistics were calculated at least three times by two independent authors in a double-blind situation. OS and the time of BM were calculated from the date of surgery to the date of BM diagnosis or to the last day of follow-up. The Kaplan-Meier method and log-rank test were used to evaluate patient survival and the cumulative risk of developing BM. Multivariate analyses for OS and BM were performed using Cox regression, and a backward-forward stepwise method was selected. Tests were two-sided, and $\mathrm{P}<0.05$ was considered to indicate a statistically significant difference.

\section{Results}

Patient characteristics. Between January 2010 and December 2013, 293 patients were diagnosed with SCLC, including 112 patients who underwent surgery. Among those 112 patients, 9 were excluded due to R1/R2 resections. An additional 12 patients were lost to follow-up, and 11 patients underwent segmentectomies. Consequently, a total of 80 patients

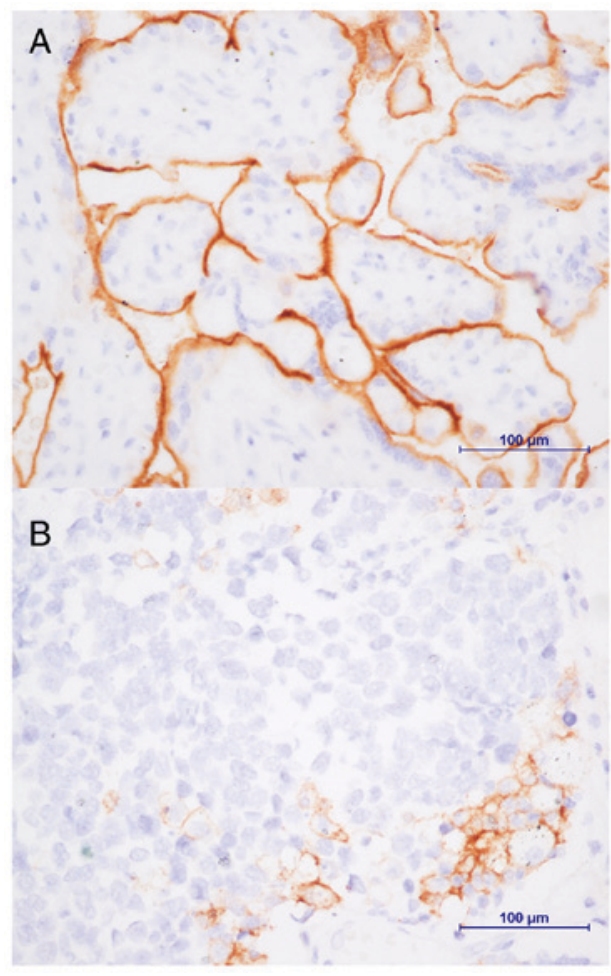

Figure 1. Immunohistochemistry analysis with SP142 antibody indicated PD-L1 expressed on the membrane of cells. (A) Positive PD-L1 staining on the cell membranes in human placenta, a strong staining intensity (3+) is presented. (B) PD-L1(+) staining was observed in 5\% of tumor cells (original magnification, x400). PD-L1, programmed death-ligand 1.

were recruited for the current study. Karnofsky performance statuses (27) following surgery were as follows: $86.3 \%$ (69/80) of patients had scores of 80-90, and $13.7 \%$ (11/80) of patients had a score of 70 . The clinical features of the patients are summarized in Table I. The median follow-up period was 52 months (range, 6-71 months). At the end of follow-up, 21.3\% (17/80) of the patients remained alive. BM was detected in a total of $27(33.8 \%)$ patients. According to the criteria of the AJCC 7th edition, no T4 or $\mathrm{N} 3$ patients were present in our patient population.

A total of 74 patients $(92.5 \%)$ underwent lobectomy, and 6 patients $(7.5 \%)$ underwent pneumonectomy. POCT was administered to $77.5 \%(62 / 80)$ of the patients, and $88.8 \%$ $(71 / 80)$ of the patients underwent PORT. Nine patients did not undergo PORT for the following reasons: 4 had poor pulmonary function and 5 had poor performance status. As PCI was not routinely recommended for postoperative patients during that period in The Department of Medical Oncology, Affiliated Hospital of Weifang Medical University, only 63.8\% (51/80) of the patients underwent PCI.

PD-L1 expression. PD-L1 expression in 80 formalin-fixed paraffin-embedded specimens was evaluated (Fig. 1). Among the cohort, staining intensities of $1+, 2+$ and $3+$ were observed in $15.0 \%(12 / 80), 28.7 \%(23 / 80)$ and $56.3 \%(45 / 80)$ of the patients, respectively. In total, PD-L1 was expressed in specimens from 52 patients (65.0\%). Among 27 patients with BM, a $3+$ staining intensity was observed in 15 patients $(55.6 \%)$, while a $1+$ intensity was observed in 4 patients $(14.8 \%)$ and a $2+$ intensity was observed in 8 patients $(29.63 \%)$. Additionally, positive PD-L1 expression was detected in 
Table II. PD-L1 expression in patients with completely resected small cell lung cancer.

\begin{tabular}{|c|c|c|c|c|}
\hline Variable & Cases $(n=80)$ & PD-L1 $\geq 5 \%$ & PD-L1 <5\% & $\mathrm{P}$-value \\
\hline Sex & & & & 0.35 \\
\hline Male & 45 & 27 & 18 & \\
\hline Female & 35 & 25 & 10 & \\
\hline Age, years & & & & 0.21 \\
\hline$\geq 60$ & 26 & 14 & 12 & \\
\hline$<60$ & 54 & 38 & 16 & \\
\hline pN stage & & & & 0.02 \\
\hline N0-1 & 56 & 41 & 15 & \\
\hline N2 & 24 & 11 & 13 & \\
\hline p-stage & & & & 0.02 \\
\hline I & 25 & 16 & 9 & \\
\hline II & 23 & 20 & 3 & \\
\hline III & 32 & 16 & 16 & \\
\hline BM status & & & & 0.47 \\
\hline Yes & 27 & 16 & 11 & \\
\hline No & 53 & 36 & 17 & \\
\hline
\end{tabular}

PD-L1, programmed death ligand 1; pN, pathological lymph node; p-stage, pathological stage; BM, brain metastasis.

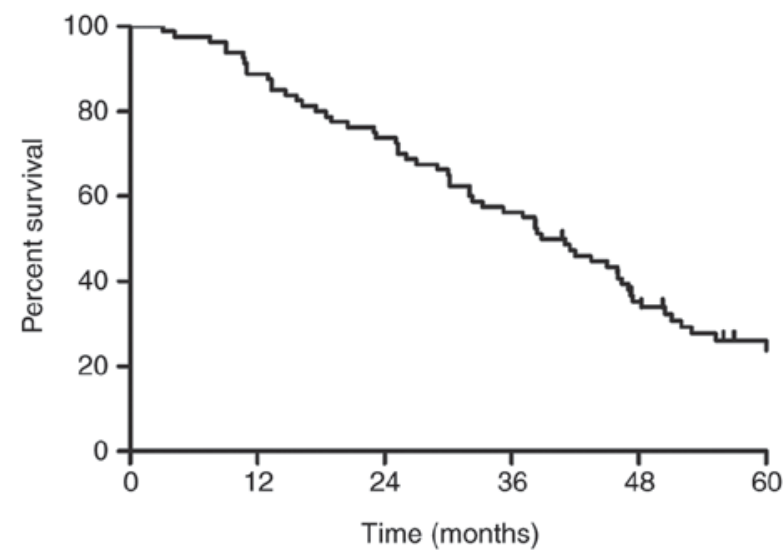

Figure 2. Kaplan-Meier plot of overall survival in 80 patients with completely resected small cell lung cancer (median survival time, 38.8 months).

16 patients $(59.3 \%)$ with BM. PD-L1 was mainly expressed in patients with pN0-1 $(51.25 \%, 41 / 80)$ and p-stage I-II disease $(45.0 \%, 36 / 80)$. The expression levels of PD-L1 in subgroups of patients with SCLC is outlined in Table II.

Survival. The survival curve for the entire group is presented in Fig. 2. The MST was 38.8 months. The 1-, 3- and 5-year survival rates were $88.8,56.3$ and $26.2 \%$, respectively. As presented in Fig. 3, the MST and 1-, 3- and 5-year survival rates were 46.4 months, $92.3,69.2$ and $33.1 \%$, respectively, among patients who were PD-L1(+) and 28.5 months, 82.1, 32.1 and $13.4 \%$, respectively, among patients who were PD-L1(-) $(\mathrm{P}=0.002)$. Univariate analysis revealed that $\operatorname{sex}(\mathrm{P}=0.034), \mathrm{N}$ stage $(\mathrm{P}=0.012)$, pathological stage $(\mathrm{P}<0.01)$, POCT $(\mathrm{P}=0.003)$, PORT $(\mathrm{P}=0.027)$, PCI $(\mathrm{P}<0.01), \mathrm{PD}-\mathrm{L} 1$ expression $(\mathrm{P}=0.001)$ and $\mathrm{BM}(\mathrm{P}<0.001)$ were associated with an increased MST of

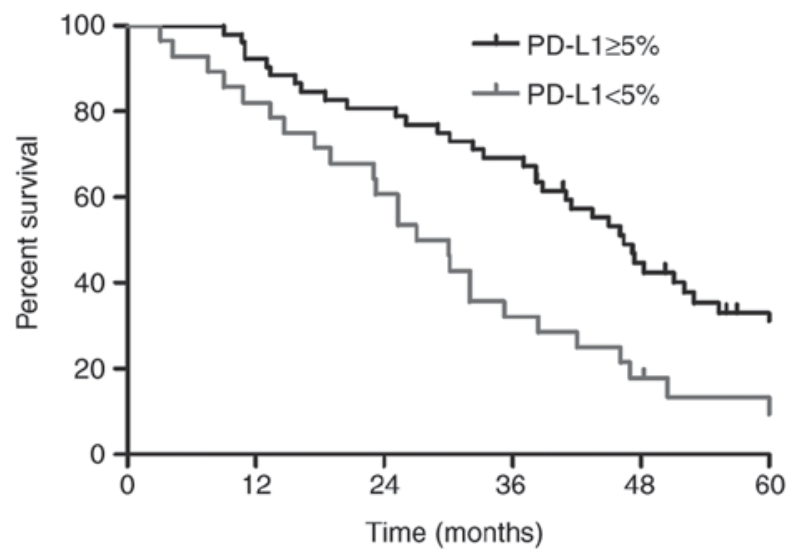

Figure 3. Kaplan-Meier plot of overall survival of patients with PD-L1(+) or PD-L1(-) tumors $(\mathrm{P}=0.002)$. PD-L1, programmed death-ligand 1.

patients with SCLC. Multivariate analysis indicated that POCT $(\mathrm{HR}=0.476, \mathrm{P}=0.023), \mathrm{PCI}(\mathrm{HR}=0.229, \mathrm{P}<0.01), \mathrm{PD}-\mathrm{L} 1$ expression $(\mathrm{HR}=0.485, \mathrm{P}=0.011)$ and $\mathrm{BM}(\mathrm{HR}=1.900, \mathrm{P}=0.026)$ were independent prognostic factors for OS (Table III).

In addition, significantly longer survival times were observed among patients without BM than among patients with BM (MST; 46.4 vs. 25.1 months; P<0.01; Fig. 4). Among the 27 patients with $\mathrm{BM}$, the survival rates between the PD-L1(+) and PD-L1 (-) groups were not significantly different $(\mathrm{P}=0.55$, data not presented).

Risk factors for BM. For the whole patient cohort, the risk of BM at 1, 3 and 5 years was 6.4, 27.5 and 40.6\%, respectively. Clinical and pathological features were evaluated to determine their predictive values for developing BM (Table IV). The risk of developing BM was associated with PD-L1 expression. As 
Table III. Univariate and multivariate analysis of the effect of prognostic factors on OS in patients with completely resected small cell lung cancer.

\begin{tabular}{|c|c|c|c|c|c|c|c|}
\hline \multirow[b]{2}{*}{ Factor } & \multicolumn{4}{|c|}{ Univariate analysis } & \multicolumn{3}{|c|}{ Multivariate analysis } \\
\hline & 1-year OS, \% & 3-year OS, \% & $\chi^{2}$ & P-value & HR & $95 \% \mathrm{CI}$ & P-value \\
\hline Sex & & & & & & & 0.896 \\
\hline Male & 86.7 & 46.7 & 4.52 & 0.034 & 0.963 & $0.543-1.707$ & \\
\hline Female & 91.4 & 68.6 & & & & & \\
\hline Age, years & & & & & & & \\
\hline$\geq 60$ & 92.3 & 46.2 & 0.065 & 0.798 & & & \\
\hline$<60$ & 87.0 & 61.1 & & & & & \\
\hline pN stage & & & & & & & 0.301 \\
\hline N0-1 & 91.1 & 64.3 & 6.285 & 0.012 & 0.594 & $0.222-1.594$ & \\
\hline $\mathrm{N} 2$ & 83.3 & 37.5 & & & & & \\
\hline p-stage & & & & & & & 0.181 \\
\hline I & 96.0 & 76.0 & 16.53 & 0.000 & 1.266 & $0.896-1.788$ & \\
\hline II & 91.3 & 65.2 & & & & & \\
\hline III & 78.1 & 34.4 & & & & & \\
\hline POCT & & & & & & & 0.023 \\
\hline Yes & 90.3 & 59.7 & 8.83 & 0.003 & 0.476 & $0.251-0.904$ & \\
\hline No & 83.3 & 44.4 & & & & & \\
\hline PORT & & & & & & & 0.116 \\
\hline Yes & 91.5 & 60.6 & 4.88 & 0.027 & 0.508 & $0.219-1.181$ & \\
\hline No & 66.7 & 22.2 & & & & & \\
\hline PCI & & & & & & & $<0.001$ \\
\hline Yes & 96.1 & 78.4 & 30.01 & $<0.001$ & 0.229 & $0.121-0.443$ & \\
\hline No & 75.9 & 17.2 & & & & & \\
\hline PD-L1 expression & & & & & & & 0.011 \\
\hline$<5 \%$ & 82.1 & 32.1 & 10.39 & 0.001 & 0.485 & $0.279-0.845$ & \\
\hline$\geq 5 \%$ & 92.3 & 69.2 & & & & & \\
\hline BM & & & & & & & 0.026 \\
\hline Yes & 81.5 & 25.9 & 20.12 & $<0.001$ & 1.900 & $1.078-3.348$ & \\
\hline No & 92.5 & 71.7 & & & & & \\
\hline
\end{tabular}

OS, overall survival; pN, pathological lymph node; p-stage, pathological stage; POCT, postoperative chemotherapy; PORT, postoperative radiotherapy; PCI, prophylactic cranial irradiation; BM, brain metastasis; PD-L1. programmed death ligand 1; CI, confidence interval.

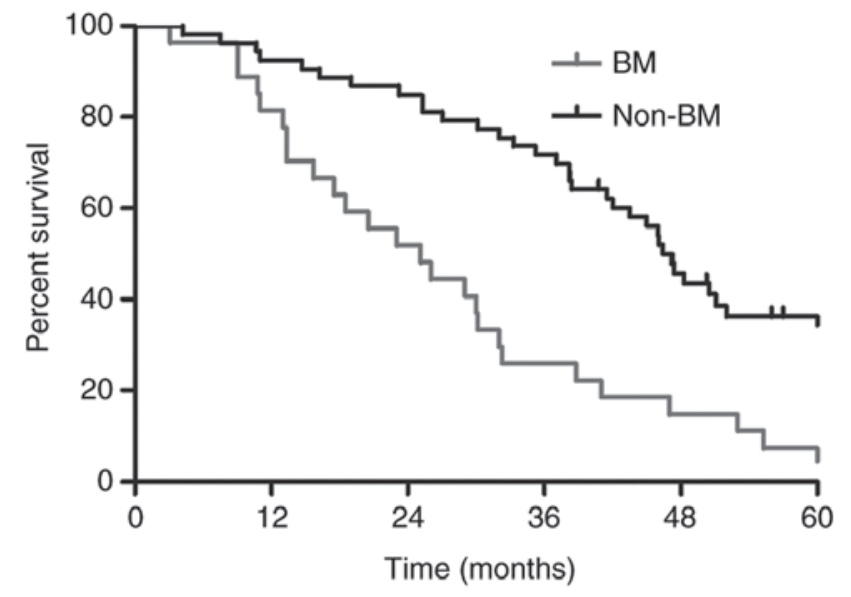

Figure 4. Kaplan-Meier plot of overall survival of patients with or without $\mathrm{BM}(\mathrm{P}<0.01)$. BM, brain metastases. presented in Fig. 5, the risk of developing BM at 1 and 3 years was 7.7 and $24.1 \%$, respectively, among PD-L1(+) patients, which was significantly lower than the 26.5 and $48.4 \%$ risk, respectively, among PD-L1(-) patients $(\mathrm{P}=0.046)$. Multivariate analysis revealed that pathological stage $(\mathrm{HR}=2.139, \mathrm{P}=0.022)$, $\mathrm{PCI}(\mathrm{HR}=0.186, \mathrm{P}<0.001)$ and $\mathrm{PD}-\mathrm{L} 1$ expression $(\mathrm{HR}=0.335$, $\mathrm{P}=0.024)$ were independent factors associated with the incidence of BM. The risk curves for the development of BM based on PCI and p-stage are presented in Fig. 6.

\section{Discussion}

In the current study, PD-L1 expression was detected by IHC with the SP142 antibody in 80 postoperative specimens from patients with SCLC. As indicated in Fig. 1, PD-L1 expression in $\geq 5 \%$ of tumor cells was observed in $65.0 \%(52 / 80)$ 
Table IV. Risk factors for developing brain metastasis in patients with completely resected small cell lung cancer.

\begin{tabular}{|c|c|c|c|c|c|c|c|}
\hline \multirow[b]{2}{*}{ Factor } & \multicolumn{4}{|c|}{ Univariate analysis } & \multicolumn{3}{|c|}{ Multivariate analysis } \\
\hline & 1-year OS,\% & 3 -year OS, \% & $\chi^{2}$ & P-value & HR & $95 \% \mathrm{CI}$ & P-value \\
\hline \multicolumn{8}{|l|}{ Sex } \\
\hline Male & 16.14 & 36.74 & 1.427 & 0.232 & & & \\
\hline Female & 11.43 & 24.51 & & & & & \\
\hline \multicolumn{8}{|c|}{ Age, years } \\
\hline$\geq 60$ & 19.23 & 32.90 & 0.04 & 0.838 & & & \\
\hline$<60$ & 11.48 & 30.75 & & & & & \\
\hline \multicolumn{8}{|l|}{ pN stage } \\
\hline N0-1 & 12.64 & 24.53 & 4.072 & 0.044 & 0.529 & $0.172-1.630$ & 0.267 \\
\hline N2 & 17.62 & 56.80 & & & & & \\
\hline p-stage & & & & & 2.139 & $1.113-4.108$ & 0.022 \\
\hline I & 8.00 & 12.38 & 10.69 & 0.001 & & & \\
\hline II & 13.04 & 22.47 & & & & & \\
\hline III & 19.97 & 56.04 & & & & & \\
\hline \multicolumn{8}{|l|}{ РOCT } \\
\hline Yes & 16.13 & 33.70 & 0.438 & 0.508 & & & \\
\hline No & 6.25 & 22.12 & & & & & \\
\hline \multicolumn{8}{|l|}{ PORT } \\
\hline Yes & 12.844 & 28.71 & 2.440 & 0.118 & & & \\
\hline No & 23.81 & 59.37 & & & & & \\
\hline \multicolumn{8}{|l|}{ PCI } \\
\hline Yes & 3.96 & 14.88 & 23.43 & $<0.001$ & 0.186 & $0.080-0.436$ & $<0.001$ \\
\hline No & 32.33 & 63.36 & & & & & \\
\hline \multicolumn{8}{|c|}{ PD-L1 expression } \\
\hline$<5 \%$ & 26.5 & 48.4 & 3.970 & 0.046 & 0.335 & $0.130-0.864$ & 0.024 \\
\hline$\geq 5 \%$ & 7.7 & 24.1 & & & & & \\
\hline
\end{tabular}

HR, hazard ratio; CI, confidence interval; OS, overall survival; $\mathrm{pN}$, pathological lymph node; $\mathrm{p}$-stage, pathological stage; POCT, postoperative chemotherapy; PORT, postoperative radiotherapy; PCI, prophylactic cranial irradiation; PD-L1, programmed death ligand 1.

of the patients. The majority of PD-L1(+) tumor cells were detected in patients with p-stage I-II (45\%) and pN0-1 disease $(51.3 \%)$. The correlation of PD-L1 expression with survival was investigated and the predictive value of PD-L1 expression for BM development was evaluated in Tables III and IV and Figs. 3 and 4. PD-L1(+) expression was a top factor associated with improved survival and a lower risk of developing BM. As depicted in Fig. 3, the MST in the PD-L1(+) group was 46.4 months, which was longer than the MST of 28.5 months in the PD-L1(-) group ( $\mathrm{P}=0.002)$. Fig. 5 depicted the risk of developing $\mathrm{BM}$ at 3 years was significantly lower in PD-L1(+) patients than in PD-L1(-) patients $(24.1 \%$ vs. $48.4 \%, \mathrm{P}=0.046)$. As depicted in Tables III and IV, multivariate analysis demonstrated that PD-L1 expression was an independent factor for survival $(\mathrm{HR}=0.485, \mathrm{P}=0.011)$ and $\mathrm{BM}(\mathrm{HR}=0.335, \mathrm{P}=0.024)$.

The PD-L1/PD-1 pathway is a negative costimulatory signal that limits effector $\mathrm{T}$ cell responses and promotes tumor evasion and intolerance (15). PD-L1 is not only expressed on the membranes of tumor cells but also in immune cells Whether PD-L1 expression on tumor cells or immune cells influences tumor progression remains controversial. Recent evidence has suggested that PD-L1 expression on tumor cells may control tumor growth $(28,29)$. PD-L1 expression in SCLC remains unclear. A retrospective analysis of PD-L1 expression in 61 pulmonary and 33 extrapulmonary cases of SCLC revealed no positive PD-L1 staining in tumor cells (19). Another study was conducted to detect PD-L1 expression in four SCLC cell lines which revealed that PD-L1 was weakly expressed on the cell surface of all four cell lines (18). However, a recent study including 249 patients with SCLC demonstrated that PD-L1 was positively expressed in $16.5 \%$ (41/249) of the tumor samples, as detected by two antibodies (SP142 and Dako $28-8$ ) with the threshold of PD-L1 positivity set as $\geq 1 \%$ cell staining (13). Additionally, a study including 102 SCLC specimens indicated $71.6 \%$ of tumor cells were PD-L1(+), which was significantly associated with limited disease SCLC (17). PD-L1(+) expression was also observed in $51.83 \%(n=83)$ SCLC specimens (20). The differences among the samples obtained, the antibodies used, the defined cut-off values and the evaluation system in these studies may contribute to the inconsistent results. Takada et al (30) evaluated PD-L1 expression in 40 surgically resected SCLC specimens by IHC using three 


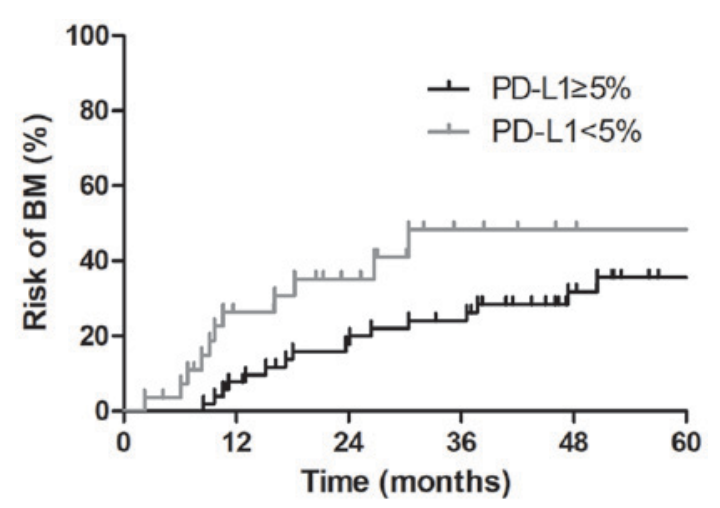

Figure 5. Kaplan-Meier plot of the risk of developing BM among patients with PD-L1(+) or PD-L1(-) tumors ( $\mathrm{P}=0.046)$. $\mathrm{PD}-\mathrm{L1}$, programmed death-ligand 1; $\mathrm{BM}$, brain metastases.
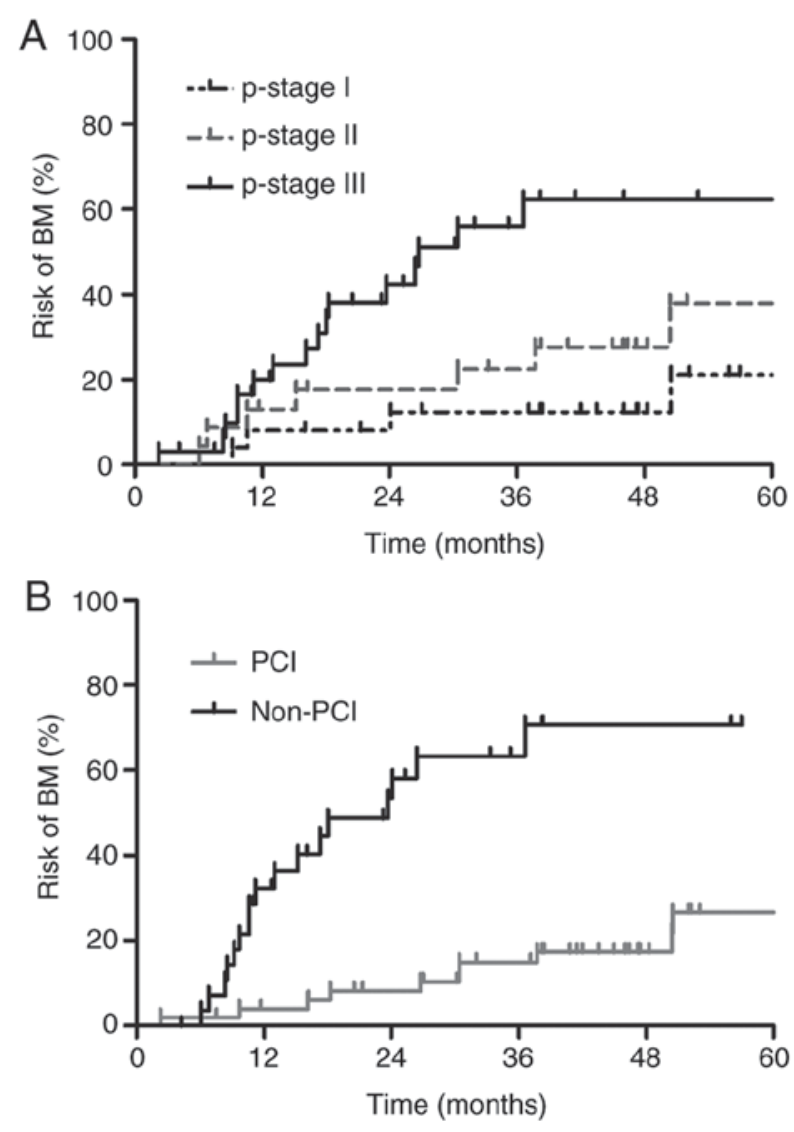

Figure 6. Kaplan-Meier plot of the risk of developing BM in patients with (A) different pathological stages and (B) treated with or without PCI. BM, brain metastases; PCI, prophylactic cranial irradiation.

different antibodies (clones E1L3 N, 28-8 and SP142) and three different evaluation methods [Allred score with range 0-8 (A, Intensity $0-3$; $\mathrm{B}$, proportion of $\mathrm{PD}-\mathrm{L} 1+$ cells $0-5$; $\mathrm{A}+\mathrm{B}=\mathrm{All}$ red score); $\geq 1 \%$ cut-off and $\geq 5 \%$ cut-off]. The results revealed that PD-L1 was positively expressed on the membranes of tumor cells, but expression levels were different (Allred score, $22.5-35 \% ; 1 \%$ cut-off, $20-32.5 \%, 5 \%$ cut-off, $15 \%$ ). In the current study, IHC was performed with the SP142 antibody to detect PD-L1 expression in postoperative specimens. The results revealed that with a cut-off of $\geq 5 \%$ staining, $\mathrm{PD}-\mathrm{L} 1$ was positively expressed in $65.0 \%$ of SCLC samples. It was noted that one study investigating PD-L1 expression in BM specimens revealed that $34.4 \%(11 / 32)$ of patients demonstrated PD-L1 expression in the membrane of tumor cells using a cut-off of $\geq 5 \%$ staining (21). At the Department of Medical Oncology, Affiliated Hospital of Weifang Medical University, where the present study was undertaken, BM diagnosis was based on imaging findings or lumbar puncture. Therefore, the prevalence of PD-L1 expression in BM tissues could not be accurately assessed.

SCLC treatment continues to rely on conventional chemotherapy, and there is not yet an effective target to improve survival. The breakthrough of anti-PD-1/PD-L1 treatment for non-small cell lung cancer and melanoma has shed light on its potential as a treatment for SCLC. Several studies have demonstrated that PD-L1(+) expression is a novel biomarker that could improve prognosis prediction among patients with SCLC $(16,17,20)$. A retrospective study including 40 patients with resected SCLC indicated an improved disease-free survival among patients with PD-L1(+) expression (16). The MST among patients with SCLC with PD-L1(+) tumors was longer than those with PD-L1(-) tumors (16.3 vs. 7.3 months, $\mathrm{P}<0.001$ ), and PD-L1 was an independent factor for survival, as demonstrated by multivariate analyses $(\mathrm{HR}=0.435$, $\mathrm{P}=0.008$ ) (17). Another study detected PD-L1 expression in 83 SCLC specimens and revealed an improved MST in patients with PD-L1(+) tumors (17.0 vs 9.0 months, $\mathrm{P}=0.018$ ) (20). Despite differences in PD-L1 antibodies, tumor tissue specimens obtained from biopsy or surgery and other clinical features among those studies, PD-L1(+) expression was consistently identified as a predictor of improved OS. In the current study, all specimens were obtained by surgery, and the prolonged survival in PD-L1(+) group further demonstrated the predictive value of PD-L1 for survival. However, no data was available to investigate the effect of PD-L1 expression on drug treatment outcome. As the patients recruited to the present study had all undergone complete resection between 2010 and 2012, no specimens were available for secondary biopsy following chemotherapy. Moreover, no checkpoint inhibitor therapy was available in China at the time of the present study.

The brain is the most common metastatic site for SCLC, and $\mathrm{BM}$ further reduces patient survival. As indicated in a previous study, patients with pathological stage I do not benefit from PCI following surgery (10); therefore, routine administration of PCI to all patients with resected SCLC may not be clinically advisable. Meanwhile, conventional chemotherapy and targeted drugs have difficulty penetrating the brain due to the blood-brain barrier. Although the correlation of PD-L1 expression has been less studied in BM from patients with SCLC, it has been initially demonstrated to be of value (24). In a large mixed cohort of 252 BM specimens, the correlation between PD-L1 expression and BM size was investigated, and the results revealed a strong negative correlation between PD-L1 expression and BM size $(\mathrm{P}=0.0016)$ (24). However, only 9 SCLC specimens were included in Harter's study. Another study including 32 SCLC specimens was conducted to detect PD-L1 expression and revealed that $75.0 \%$ of $\mathrm{BM}$ specimens expressed PD-L1, with $34.4 \%$ of cases demonstrating PD-L1 expression in $\geq 5 \%$ of the tumor cells; furthermore, PD-L1 expression in tumor cells was not correlated with survival $(\mathrm{P}=0.662)(21)$. In the present study, the risk of developing BM in the PD-L1(+) group was 
significantly lower than that in the PD-L1(-) group. Regardless of the variations between studies, the data suggested that the PD-L1 pathway serves an important role in BM in SCLC and reveal a positive effect of PD-L1 positivity on clinical outcomes.

Several limitations can be attributed to this retrospective study. As previously mentioned, only $38.2 \%$ (112/293) of patients with SCLC underwent surgery within four years at the Department of Medical Oncology, Affiliated Hospital of Weifang Medical University, where the present study was undertaken. According to the criteria of enrolment, the number of eligible patients was limited to 80 . For this retrospective study however, the data from this small cohort is sufficient to explain certain findings. In addition, there were no approved antibodies for detecting PD-L1 expression at the time of the study. On the other hand, SP142 was more readily available than other antibodies. In addition, as the present study was a preliminary exploratory study, research funding was identified as a limiting factor. Furthermore, the initial objective was to investigate the expression of PD-L1 in resectable SCLC. Following completion of the preliminary IHC result analysis, the findings of the present study were presented. We have performed animal experiments to establish a SCLC brain metastasis model and investigated the role of PD-L1/PD-1 and other immune-related molecules in survival and response to radiotherapy and checkpoint inhibitor treatment.

PD-L1 is commonly expressed on the membranes of SCLC cells. PD-L1(+) expression is associated with reduced risk of $\mathrm{BM}$ and is predictive of improved survival, which suggests that checkpoint inhibition therapy at an optimal time in the course of the disease may further prolong survival. Considering these results and the small sample size in this retrospective study, further large-scale and/or randomized studies are urgently required.

\section{Acknowledgements}

Not applicable.

\section{Funding}

The present study was supported by the Scientific Research Project of Weifang Health Bureau to Jin Liu (grant no. 2014013).

\section{Availability of data and materials}

The datasets used and/or analyzed in the present study are available from the corresponding author upon reasonable request.

\section{Authors' contributions}

JL performed the statistical analysis and drafted the manuscript, ZL helped collect the data and revise the manuscript, XS designed the study, JL collected the data and WW performed the statistical analysis and revised the manuscript. All authors have read and approved the final manuscript.

\section{Ethics approval and consent to participate}

The present study was approved by the Institutional Review Board or Ethics Committee of the Affiliated Hospital of
Weifang Medical University. Written informed consent was obtained from every patient and/or their legal guardian prior to inclusion in the present study.

\section{Consent for publication}

Consent for publication was obtained from all participants and/or their legal guardian.

\section{Competing interests}

The authors declare that they have no competing interests.

\section{References}

1. Siegel RL, Miller KD and Jemal A: Cancer statistics, 2015. CA Cancer J Clin 65: 5-29, 2015.

2. Govindan R, Page N, Morgensztern D, Read W, Tierney R, Vlahiotis A, Spitznagel EL and Piccirillo J: Changing epidemiology of small-cell lung cancer in the United States over the last 30 years: Analysis of the surveillance, epidemiologic, and end results database. J Clin Oncol 24: 4539-4544, 2006.

3. Bayman NA, Sheikh H, Kularatne B, Lorigan P, Blackhall F, Thatcher $\mathrm{N}$ and Faivre-Finn C: Radiotherapy for small-cell lung cancer-Where are we heading? Lung Cancer 63: 307-314, 2009.

4. Turrisi AT III, Kim K, Blum R, Sause WT, Livingston RB, Komaki R, Wagner H, Aisner S and Johnson DH: Twice-daily compared with once-daily thoracic radiotherapy in limited small-cell lung cancer treated concurrently with cisplatin and etoposide. N Engl J Med 340: 265-271, 1999.

5. Jeremic B, Shibamoto Y, Nikolic N, Milicic B, Milisavljevic S, Dagovic A, Aleksandrovic J and Radosavljevic-Asic G: Role of radiation therapy in the combined-modality treatment of patients with extensive disease small-cell lung cancer: A randomized study. J Clin Oncol 17: 2092-2099, 1999.

6. Veronesi G, Bottoni E, Finocchiaro G and Alloisio M: When is surgery indicated for small-cell lung cancer? Lung Cancer 90: 582-589, 2015.

7. Socha J and Kępka L: Prophylactic cranial irradiation for small-cell lung cancer: How, when and for whom? Expert Rev Anticancer Ther 12: 505-517, 2012.

8. Seute T, Leffers P, ten Velde GP and Twijnstra A: Neurologic disorders in 432 consecutive patients with small cell lung carcinoma. Cancer 100: 801-806, 2004.

9. Komaki R, Cox JD and Whitson W: Risk of brain metastasis from small cell carcinoma of the lung related to length of survival and prophylactic irradiation. Cancer Treat Rep 65: 811-814, 1981.

10. Zhu H, Guo H, Shi F, Zhu K, Luo J, Liu X, Kong L and Yu J: Prophylactic cranial irradiation improved the overall survival of patients with surgically resected small cell lung cancer, but not for stage I disease. Lung Cancer 86: 334-338, 2014.

11. Sunshine JC, Nguyen PL, Kaunitz GJ, Cottrell TR, Berry S, Esandrio J, Xu H, Ogurtsova A, Bleich KB, Cornish TC, et al: PD-L1 expression in melanoma: A quantitative immunohistochemical antibody comparison. Clin Cancer Res 23: 4938-4944, 2017.

12. He Y, Rozeboom L, Rivard CJ, Ellison K, Dziadziuszko R, Yu H, Zhou C and Hirsch FR: PD-1, PD-L1 protein expression in non-small cell lung cancer and their relationship with tumor-infiltrating lymphocytes. Med Sci Monit 23: 1208-1216, 2017.

13. Yu H, Batenchuk C, Badzio A, Boyle TA, Czapiewski P, Chan DC, Lu X, Gao D, Ellison K, Kowalewski AA, et al: PD-L1 expression by two complementary diagnostic assays and mRNA in situ hybridization in small cell lung cancer. J Thorac Oncol 12: 110-120, 2017.

14. Dong H, Strome SE, Salomao DR, Tamura H, Hirano F, Flies DB, Roche PC, Lu J, Zhu G, Tamada K, et al: Tumor-associated B7-H1 promotes T-cell apoptosis: A potential mechanism of immune evasion. Nat Med 8: 793-800, 2002.

15. Keir ME, Butte MJ, Freeman GJ and Sharpe AH: PD-1 and its ligands in tolerance and immunity. Annu Rev Immunol 26: 677-704, 2008 
16. Toyokawa G, Takada K, Haratake N, Takamori S, Akamine T, Katsura M, Fujishita T, Shoji F, Okamoto T, Oda Y and Maehara Y: Favorable disease-free survival associated with programmed death ligand 1 expression in patients with surgically resected small-cell lung cancer. Anticancer Res 36: 4329-4336, 2016.

17. Ishii H, Azuma K, Kawahara A, Yamada K, Imamura Y, Tokito T, Kinoshita T, Kage M and Hoshino T: Significance of programmed cell death-ligand 1 expression and its association with survival in patients with small cell lung cancer. J Thorac Oncol 10: 426-430, 2015.

18. Yamane H, Isozaki H, Takeyama M, Ochi N, Kudo K, Honda Y, Yamagishi T, Kubo T, Kiura K and Takigawa N: Programmed cell death protein 1 and programmed death-ligand 1 are expressed on the surface of some small-cell lung cancer lines. Am J Cancer Res 5: 1553-1557, 2015.

19. Schultheis AM, Scheel AH, Ozretić L, George J, Thomas RK, Hagemann T, Zander T, Wolf J and Buettner R: PD-L1 expression in small cell neuroendocrine carcinomas. Eur J Cancer 51: 421-426, 2015.

20. Miao L, Lu Y, Xu Y, Zhang G, Huang Z, Gong L and Fan Y: PD-L1 and c-MET expression and survival in patients with small cell lung cancer. Oncotarget 8: 53978-53988, 2017.

21. Berghoff AS, Ricken G, Wilhelm D, Rajky O, Widhalm G, Dieckmann K, Birner P, Bartsch R and Preusser M: Tumor infiltrating lymphocytes and PD-L1 expression in brain metastases of small cell lung cancer (SCLC). J Neurooncol 130: 19-29, 2016

22. Yan F, Pang J, Peng Y, Molina JR, Yang P and Liu S: Elevated cellular PD1/PD-L1 expression confers acquired resistance to cisplatin in small cell lung cancer cells. PLoS One 11: e0162925, 2016.

23. George J, Saito M, Tsuta K, Iwakawa R, Shiraishi K, Scheel AH, Uchida S, Watanabe SI, Nishikawa R, Noguchi M, et al: Genomic amplification of CD274 (PD-L1) in small-cell lung cancer. Clin Cancer Res 23: 1220-1226, 2017.
24. Harter PN, Bernatz S, Scholz A, Zeiner PS, Zinke J, Kiyose M, Blasel S, Beschorner R, Senft C, Bender B, et al: Distribution and prognostic relevance of tumor-infiltrating lymphocytes (TILs) and PD-1/PD-L1 immune checkpoints in human brain metastases. Oncotarget 6: 40836-40849, 2015.

25. Kligerman $\mathrm{S}$ and Abbott G: A radiologic review of the new TNM classification for lung cancer. AJR Am J Roentgenol 194: 562-573, 2010

26. Liang SC, Latchman YE, Buhlmann JE, Tomczak MF, Horwitz BH, Freeman GJ and Sharpe AH: Regulation of PD-1, PD-L1 and PD-L2 expression during normal and autoimmune responses. Eur J Immunol 33: 2706-2716, 2003.

27. Schag CC, Heinrich RL and Ganz PA: Karnofsky performance status revisited: Reliability, validity, and guidelines. J Clin Oncol 2: 187-193, 1984.

28. Lau J, Cheung J, Navarro A, Lianoglou S, Haley B, Totpal K, Sanders L, Koeppen H, Caplazi P, McBride J, et al: Tumour and host cell PD-L1 is required to mediate suppression of anti-tumour immunity in mice. Nat Commun 8: 14572, 2017.

29. Juneja VR, McGuire KA, Manguso RT, LaFleur MW, Collins N, Haining WN, Freeman GJ and Sharpe AH: PD-L1 on tumor cells is sufficient for immune evasion in immunogenic tumors and inhibits CD8 T cell cytotoxicity. J Exp Med 214: 895-904, 2017.

30. Takada K, Toyokawa G, Okamoto T, Akamine T, Takamori S, Katsura M, Fujishita T, Shoji F, Oda Y and Maehara Y: An immunohistochemical analysis of PD-L1 protein expression in surgically resected small cell lung cancer using different antibodies and criteria. Anticancer Res 36: 3409-3412, 2016.

This work is licensed under a Creative Commons Attribution-NonCommercial-NoDerivatives 4.0 International (CC BY-NC-ND 4.0) License. 